\title{
Evolución del complejo agroalimentario de Navarra. Análisis a partir de las tablas input-output de 1980 y 1995
}

\author{
Belén Iráizoz Apezteguía $^{1}$, Manuel Rapún Gárate ${ }^{2}$
}

RESUMEN: En este trabajo se estudia el complejo agroalimentario de Navarra a partir de la información suministrada por las Tablas Input-Output de 1980 y 1995. Se trata de analizar la evolución de la importancia relativa de las ramas pertenecientes al complejo y de las relaciones que se establecen entre las mismas. Los indicadores utilizados para el estudio son los coeficientes de Streit, Chenery y Watanabe, Rasmussen y los multiplicadores de renta y empleo. Del análisis de estos indicadores se desprende el importante papel que tienen la agricultura y la fabricación de conservas vegetales en el entorno de la economía regional.

PALABRAS CLAVE: complejo agroalimentario, análisis Input-Output, Navarra CóDIGOS JEL: O13, Q12

\section{Evolution of the Agrifood complex in Navarra. An analysis using input-output tables 1980-1995.}

\begin{abstract}
This paper analyses the agrofood system in Navarra (Spain) using the InputOutput data for 1980 and 1995. The aim is to analyse the evolution and changes taking place between the economic activities in the region. Streit, Chenery-Watanabe and Rasmussen's indicators are constructed. As is usual, we have also calculated the inconme and employment multipliers. This indicators show the very important role off the agriculture and the vegetables canning industry in the regional economy.
\end{abstract}

KEY WORDS: agrofood sector, Input-Output analysis, Navarra

\footnotetext{
1 Departamento de Economía. Universidad Pública de Navarra. Campus de Arrosadía, 31006 Pamplona (Navarra). T.: 948169345 iraizoz@si.unavarra.es

2 Departamento de Economía. Universidad Pública de Navarra. Campus de Arrosadía, 31006 Pamplona (Navarra).T.: 948169357 mrapun@si.unavarra.es
} 



\section{INTRODUCCIÓN}

El objetivo de este trabajo consiste en analizar la evolución de la estructura productiva del Complejo Agroalimentario (CAA, en adelante) de Navarra. La información utilizada procede de las tablas Input-Output de Navarra de los años 1980 y 1995 (TION-80 y TION-95, en adelante).

El interés en realizar este estudio se basa en que tradicionalmente el CAA ha tenido una importancia significativa en el conjunto económico regional. Basta señalar que en 1980 representaba el 14,8\% del Valor Añadido Bruto, el 19,9\% de la producción a precios salida de fábrica y el 22,5\% de las exportaciones regionales ${ }^{3}$. A partir de las TION-95 se puede inferir que ha perdido cierta relevancia ya que representa el 9,05\% del Valor Añadido Bruto, el 12\% de la producción efectiva y el $18 \%$ de las exportaciones al exterior de la región. En este contexto, Navarra es una región que en el entorno nacional está especializada en el sector agroalimentario (Rodríguez, 1998).

Las TION han sido homogeneizadas con un nivel de desagregación de 51 ramas de actividad $^{4}$. En dichas tablas el CAA, que incluye la producción primaria y las industrias transformadoras de alimentos, está integrado por las siguientes ramas ${ }^{5}$ : Agricultura, Ganadería, Caza, silvicultura y pesca, Industria de productos cárnicos, Fabricación de conservas vegetales, Industria de productos lácteos, Pan, bollería y galletas, Otras industrias de alimentación, Industria vinícola y Otras industrias de bebida y tabaco. Las tres primeras hacen referencia al sector primario y las siete restantes se corresponden con la industria agroalimentaria (IAA, en adelante).

El enfoque de análisis utilizado se basa en las aportaciones de Malassis (1979) que se sirve de las TIO como base fundamental de información estadística económica. Este tipo de información permite conocer las relaciones de compraventa y medir el grado de integración que existe entre los sectores que forman parte del sistema. Dado que en nuestro caso se dispone de dos TIO, nos vamos a centrar en los cambios que se han producido en dichas relaciones. Ahora bien, se debe señalar que debido al amplio período de tiempo que media

\footnotetext{
3 En el análisis consideramos que las exportaciones e importaciones engloban las transacciones que tienen su destino o su origen fuera del territorio navarro.

4 Las TION proceden del CD-ROM "Matrices derivadas de las Tablas Input-Output para 1980 y 1995 " del Instituto de Estadística de Navarra.

5 La correspondencia entre estas ramas de actividad y las derivadas de la Clasificación Nacional e Actividades Económicas de 1993 puede verse en Gobierno de Navarra (1997).
} 
entre las dos tablas, se presentan algunos problemas a la hora de comparar los resultados obtenidos a partir de las mismas.

En primer lugar destaca el hecho de que no se dispone de las tablas valoradas a precios constantes. Esto implica que no va a ser posible diferenciar los cambios producidos por variaciones en los precios de los derivados de cambios tecnológicos.

En segundo lugar, la metodología utilizada en la elaboración de las tablas se ha modificado. Así, ha cambiado el sistema de valoración de los flujos, como consecuencia de la incorporación del IVA. De forma que en 1980 la matriz de relaciones intersectoriales y la producción se valora a precios salida de fábrica incluyendo los impuestos sobre la producción netos de subvenciones, mientras que en 1995 la valoración se realiza a precios salida de fábrica sin incluir el IVA. De la misma manera ha variado la valoración de las importaciones, que en 1995 no incorporan el IVA.

El número de ramas de actividad considerado también ha variado, ya que la TION-80 consideraba 75 ramas que han sido agregadas en las 51 presentes en la TION-95. Por lo tanto, también se deben tener presentes las limitaciones derivadas de dicho proceso de agregación.

Otra diferencia metodológica relevante consiste en que en la TION-80 no se considera la producción imputada de servicios bancarios (PISB), mientras que sí la incorpora la TION-95. Dado que esta circunstancia produce diferencias relevantes en los coeficientes obtenidos, en este trabajo se ha optado por eliminar dicha PISB de la TION-95, de forma que los coeficientes obtenidos consideran como producción efectiva de la rama de intermediarios financieros solamente la producción de servicios destinados a la venta.

Este enfoque ha sido utilizado frecuentemente para estudiar el CAA español. Entre las aportaciones que realizan un análisis de su evolución destacan las de Titos y De Haro (1983) que estudian la evolución del CAA y lo comparan con la de 5 países europeos. Enciso y Sabaté (1995a) se centran en el estudio de la evolución del CAA español durante la década de los ochenta. López (1995) analiza los cambios estructurales de la IAA en el mismo período. Titos et al. (1996) estudian el cambio estructural en el sistema agroalimentario entre 1970 y 1988.

También se ha aplicado al estudio de complejos agroalimentarios regionales. Así, De La Grana y Azaceta (1990) analizan el CAA de Euskadi en 1985, Pérez y Feijoo (1993) estudian la estructura del CAA aragonés en 1990, Artis et al. (1994) se centran en el CAA catalán en 1987, Enciso y Sabaté (1995b) comparan el CAA catalán con el español en 1987, Titos (1995) analiza el CAA andaluz en 1990, De Pablo y Céspedes (1996) completan el trabajo anterior 
comparando los resultados con los obtenidos a partir de la TIO de 1980, García y Enguídanos (1999) abordan el estudio del sector agroalimentario valenciano en 1990, e Iráizoz y Rapún (1999) que analizan el CAA de Navarra en 1995.

El contenido del trabajo se estructura de acuerdo al siguiente esquema. En primer lugar, y para cada uno de los años considerados, se estudia la importancia relativa del CAA y sus diferentes ramas en el entorno de la economía regional. A continuación, se analiza la evolución de las relaciones de compraventa que se establecen entre las ramas agroalimentarias con el fin de constatar cómo ha variado la capacidad que tienen de impulsar y/o recibir impulsos del resto de ramas. Posteriormente, se estudian los cambios producidos en estos quince años en la capacidad para generar renta y empleo en el territorio navarro. Finalmente, se presentan las conclusiones más relevantes del trabajo.

\section{LAS RAMAS PRODUCTIVAS EN EL CAA DE NAVARRA}

La primera visión del CAA de Navarra se obtiene a partir de las principales magnitudes macroeconómicas que caracterizan al mismo, así como a cada una de las ramas que lo integran.

De acuerdo a la información recogida en el Cuadro 1, el total de recursos de productos agroalimentarios en 1995 se cifra en algo más de 470.000 millones de pesetas, correspondiendo el $64 \%$ a la producción interna y el $34 \%$ aportado por las importaciones. En 1980, estas cifras alcanzaban los valores del $69 \%$ y $31 \%$ respectivamente.

Del total de recursos agroalimentarios disponibles en 1995, el 37\% son utilizados como inputs intermedios en el territorio navarro, mientras que a la demanda final interior se destinan el $23 \%$, cifra inferior al $40 \%$ que se exporta fuera de la región. Estas cifras no han variado significativamente respecto a las existentes en 1980.

En 1995, el saldo de la balanza comercial es excedentario para los productos del CAA en 28.000 millones de pesetas, presentando un grado de cobertura de las exportaciones sobre las importaciones del 117,4\%, que para la economía navarra es del 101,8\%. En este sentido cabe destacar la sensible disminución del grado de cobertura, dado que en 1980 alcanzaba el valor del $137 \%$.

El 9,05\% de la renta generada en la economía (VABpm) corresponde al CAA, para lo cual emplean al $12 \%$ de los trabajadores regionales, por lo que en principio el CAA presenta una productividad aparente del trabajo inferior a la media regional, circunstancia que también se producía en 1980. 
Cuadro 1. Complejo agroalimentario y economía navarra. 1980-1995.

\begin{tabular}{lcccccc}
\hline & \multicolumn{3}{c}{ CAA 1980 } & \multicolumn{3}{c}{ CAA 1995 } \\
\hline & Valor (1) & $\begin{array}{c}\text { \% Recur. } \\
\text { Totales }\end{array}$ & $\begin{array}{c}\text { \% Economía } \\
\text { de Navarra }\end{array}$ & Valor (1) & $\begin{array}{c}\text { \% Recur. } \\
\text { Totales }\end{array}$ & $\begin{array}{c}\text { \% Economía de } \\
\text { Navarra }\end{array}$ \\
\hline VABpm & $32.361,6$ & 24,50 & 14,79 & $95.784,1$ & 20,28 & 9,05 \\
Producción Efectiva & $90.924,3$ & 68,82 & 19,88 & $302.723,5$ & 64,10 & 11,93 \\
Importaciones Equivalen. & $40.668,9$ & 30,78 & 16,94 & $161.174,7$ & 34,13 & 15,69 \\
Recursos Totales & $132.115,9$ & 100 & 18,90 & $472.264,4$ & 100 & 13,04 \\
Outputs Intermedios & $47.689,9$ & 36,10 & 19,86 & $173.504,1$ & 36,74 & 12,15 \\
Demanda Final Interior & $28.725,6$ & 21,74 & 13,57 & $109.518,4$ & 23,19 & 9,54 \\
Exportaciones & $55.700,4$ & 42,13 & 22,53 & $189.241,9$ & 40,07 & 18,09 \\
Empleo & 30.725 & - & 17,83 & 21.732 & - & 12,01 \\
\hline $\begin{array}{l}\text { Fuente: TION-80 y TION-95. (1) Las magnitudes están expresadas en millones de pesetas corrientes, excepto el empleo que viene } \\
\text { expresado como número de personas ocupadas. }\end{array}$ & \multicolumn{5}{c}{}
\end{tabular}

Con el fin de estudiar la importancia de cada rama en el CAA, el Cuadro 2 expone la participación de cada una de ellas en la formación de las magnitudes más relevantes. Se observa cómo el 45\% del Valor Añadido Bruto a precios de mercado (VABpm) del CAA es aportado por las actividades primarias y el 55\% restante por la industria agroalimentaria. Por ramas destacan las de agricultura, ganadería y fabricación de conservas vegetales, con más del $10 \%$ del VABpm total.

Sin embargo, el peso del sector primario en la producción efectiva del CAA es bastante menor $(31 \%)$, aunque siguen destacando las ramas de agricultura y de ganadería con aportaciones superiores al $10 \%$. Entre las ramas pertenecientes a la IAA destacan por sus mayores aportaciones las ramas de fabricación de conservas vegetales y otras industrias de alimentación.

En relación a las exportaciones, el $81 \%$ de las mismas corresponden a la IAA, destacando las actividades de fabricación de conservas vegetales que representan casi un 30\%. Respecto a las importaciones, sobresalen los productos agroalimentarios transformados (61\%). En todo caso, los productos importados con mayor participación son los incluidos en la rama de agricultura.

Para analizar cómo ha variado la composición del CAA a lo largo de estos quince años, se han calculado dos índices de variación ${ }^{6}$ de la especialización relativa (IESPREL) de las ramas en el CAA, utilizando de forma alternativa el VABpm y la producción efectiva.

\footnotetext{
6 Las expresiones utilizadas para su cálculo son las siguientes:

$\operatorname{IESPREL}_{\mathrm{i}}^{\mathrm{VAB}}=\left(\mathrm{VAB}_{\mathrm{i}} / \mathrm{VAB}_{\mathrm{CAA}}\right)_{95} /\left(\mathrm{VAB}_{\mathrm{i}} / \mathrm{VAB}_{\mathrm{CAA}}\right)_{80}$ y IESPREL $\mathrm{IE}_{\mathrm{i}}^{\mathrm{PE}}=\left(\mathrm{PE}_{\mathrm{i}} / \mathrm{PE}_{\mathrm{CAA}}\right)_{95} /\left(\mathrm{PE}_{\mathrm{i}} / \mathrm{PE}_{\mathrm{CAA}}\right)$.
} 
Cuadro 2. Importancia de cada rama en el CAA en 1995 (1).

\begin{tabular}{lccrrrrrrr}
\hline & VAB & PE & EXP & IMP & \multicolumn{1}{l}{ IESPREL } & IESPREL & VARPEXP VARPIMP \\
& & & & & & $(3)$ & \\
\hline 1. Agricultura & 28,3 & 16,1 & 3,6 & 22,0 & 0,62 & 0,63 & 0,26 & 1,20 \\
2. Ganadería & 12,1 & 13,6 & 14,7 & 6,2 & 1,12 & 0,92 & 1,88 & 1,44 \\
3. Caza, silvicultura y pesca & 4,9 & 1,6 & 0,3 & 10,4 & 0,99 & 0,74 & 2,81 & 1,15 \\
Agricultura, ganadería y sil. & $\mathbf{4 5 , 2}$ & $\mathbf{3 1 , 3}$ & $\mathbf{1 8 , 5}$ & $\mathbf{3 8 , 5}$ & $\mathbf{0 , 7 4}$ & $\mathbf{0 , 7 4}$ & $\mathbf{0 , 8 3}$ & $\mathbf{1 , 2 6}$ \\
5. Industria de productos cárnicos & 6,7 & 9,9 & 9,3 & 18,7 & 1,11 & 0,91 & 1,23 & 1,43 \\
6. Fabricación de conservas veget. & 14,3 & 20,2 & 29,2 & 4,3 & 1,99 & 2,27 & 0,94 & 1,45 \\
7. Industria de productos lácteos & 2,4 & 4,2 & 6,2 & 6,7 & 0,57 & 0,51 & 1,35 & 2,59 \\
8. Pan, bollería y galletas & 6,7 & 4,6 & 2,9 & 2,3 & 1,74 & 1,08 & 0,64 & 0,72 \\
9. Otras industrias de aliment. & 7,3 & 12,4 & 10,1 & 13,2 & 0,69 & 0,66 & 0,62 & 0,61 \\
10. Industria vinícola & 8,6 & 7,6 & 9,4 & 1,3 & 2,47 & 2,31 & 1,01 & 0,37 \\
11. Otras indust. bebida y tabaco & 8,8 & 9,6 & 14,3 & 14,9 & 2,47 & 3,02 & 1,21 & 1,19 \\
Industria Agroalimentaria & $\mathbf{5 4 , 7}$ & $\mathbf{6 8 , 6}$ & $\mathbf{8 1 , 5}$ & $\mathbf{6 1 , 5}$ & $\mathbf{1 , 4 1}$ & $\mathbf{1 , 2 0}$ & $\mathbf{1 , 0 1}$ & $\mathbf{0 , 9 6}$ \\
Complejo Agroalimentario & $\mathbf{1 0 0}$ & $\mathbf{1 0 0}$ & $\mathbf{1 0 0}$ & $\mathbf{1 0 0}$ & $\mathbf{1}$ & $\mathbf{1}$ & $\mathbf{1 , 0 2}$ & $\mathbf{1 , 0 7}$ \\
\hline
\end{tabular}

Fuente: TION-80 y TION-95. (1) VAB: Valor Añadido Bruto a precios de mercado. PE: Producción efectiva. EXP: Exportaciones. IMP: Importaciones equivalentes. IESPREL: Variación en la especialización relativa. VARPEXP: Variación en la propensión exportadora. VARPIMP: Variación en la propensión importadora. (2) Calculado utilizando el VABpm. (3) Calculado con la producción efectiva.

A la vista de los mismos, se puede señalar que la agricultura, la industria de productos lácteos y las otras industrias de alimentación han perdido importancia en el conjunto del CAA. Sin embargo, las industrias de fabricación de conservas vegetales, la industria vinícola y las otras industrias de bebidas y tabaco han aumentado sensiblemente su participación en las macromagnitudes del CAA regional.

También se han producido cambios en la propensión exportadora e importadora ${ }^{7}$ (VARPEXP y VARPIMP) de las ramas pertenecientes al CAA. Así, en cuanto a la primera, destaca el aumento producido para las ramas de ganadería y la industria de productos lácteos, y la disminución producida en la rama de agricultura. En cuanto a la propensión importadora, se observa el importante aumento producido para los productos correspondientes a las industrias lácteas, y la sensible disminución para aquellos correspondientes a las industrias vinícolas.

Una vez contextualizado el CAA en la economía de Navarra, se presentan los resultados de los análisis de las TION-80 y TION-95 para las diez ramas de actividad consideradas. Pero antes debemos hacer una matización en cuanto al uso de las matrices, ya que dichos análisis se pueden realizar teniendo en cuenta la matriz de transacciones interindustriales interiores o

7 En este caso las expresiones son: 
totales. En nuestro caso, y dado que nuestro interés se centra en realizar una valoración de los efectos intersectoriales sobre la producción interior de la economía navarra, siguiendo las recomendaciones realizadas en la literatura ${ }^{8}$, nos vamos a centrar en el análisis de los resultados obtenidos a partir de las matrices interiores, aunque los indicadores obtenidos se han calculado teniendo en cuenta las matrices de coeficientes técnicos interiores y los totales.

\section{ARTICULACIÓN DEL TEJIDO PRODUCTIVO DEL CAA}

La medición del estudio de las relaciones de dependencia entre los sectores se inicia con las denominadas ligazones o eslabonamientos, conceptos introducidos por Hirschman (1958) en los estudios de desarrollo. La dirección y el peso de las ligazones sectoriales indican la capacidad potencial de un sector para estimular a otros, por lo que la concentración de recursos en aquellas actividades con mayores ligazones impulsará más rápidamente el crecimiento de la producción, renta y empleo que la dedicación de dichos recursos a otras actividades (Del Castillo y García, 1990).

En la literatura se han desarrollado diferentes propuestas con el fin de cuantificar estos eslabonamientos. En nuestro caso, se van a utilizar los coeficientes de Streit, los coeficientes de Chenery y Watanabe, los efectos absorción y difusión y los coeficientes de Rasmussen.

\subsection{Coeficientes de Streit}

Los denominados coeficientes de Streit (1969) pretenden cuantificar las relaciones que se establecen entre cada par de ramas, y se especifican de la siguiente manera:

$$
\operatorname{CES}_{\mathrm{ij}}=\frac{1}{4}\left(\frac{\mathrm{x}_{\mathrm{ij}}}{\sum_{\mathrm{j}} \mathrm{x}_{\mathrm{ij}}}+\frac{\mathrm{x}_{\mathrm{ij}}}{\sum_{\mathrm{i}} \mathrm{x}_{\mathrm{ij}}}+\frac{\mathrm{x}_{\mathrm{ji}}}{\sum_{\mathrm{j}} \mathrm{x}_{\mathrm{ji}}}+\frac{\mathrm{x}_{\mathrm{ji}}}{\sum_{\mathrm{i}} \mathrm{x}_{\mathrm{ji}}}\right)
$$

donde $\mathrm{x}_{\mathrm{ij}}$ representa el valor de los consumos intermedios que la rama $j$ hace de productos de la rama $i$. El cálculo de estos coeficientes ${ }^{9}$ permite seleccionar las ramas polarizantes, es decir, aquéllas a las que va una parte importante de las salidas intermedias de otras ramas y de las que procede una parte importante de los inputs intermedios utilizados por otras ramas. Serían ramas que agrupan en su entorno a otras como oferentes o demandantes de inputs intermedios.

\footnotetext{
VARPEXP $_{\mathrm{i}}=\left(\mathrm{EXP}_{\mathrm{i}} / \mathrm{PE}_{\mathrm{i}}\right)_{95} /\left(\mathrm{EXP}_{\mathrm{i}} / \mathrm{PE}_{\mathrm{i}}\right)_{80}$ y VARPIMP $\mathrm{i}=\left(\mathrm{IMP}_{\mathrm{i}} / \mathrm{DTINT}_{\mathrm{i}}\right)_{95} /\left(\mathrm{IMP}_{\mathrm{i}} / \mathrm{DTINT}_{\mathrm{i}}\right)_{80}$. 8 Véanse, por ejemplo, Del Castillo y Martínez (1986), Del Castillo y García (1990) y Pulido y Fontela (1993).

9 Se trata de calcular la media aritmética de las dos ligazones de oferta y las dos ligazones de demanda que se establecen entre cada par de ramas.
} 
Ahora bien, estos coeficientes presentan algunas limitaciones, siendo las más relevantes que priman a las ramas que concentran la oferta y la demanda por insignificantes que sean en términos absolutos y que se tratan igual las relaciones hacia atrás que las relaciones hacia adelante (Cuadrado y Mancha, 1984).

Dado el número de ramas consideradas, en el Cuadro 3 se presentan solamente los coeficientes de Streit de las ramas pertenecientes al CAA tomando como umbral de relevancia el valor 0,1 .

Si hacemos referencia a los coeficientes obtenidos a partir de las TION-95, las ramas con mayor número de coeficientes relevantes se corresponden con las incluidas en el sector primario. Así, la agricultura es la que presenta mayor número de ligazones relevantes, siendo en su mayoría con ramas del CAA, aunque también las presenta con la industria química y la de construcción de maquinaria por la demanda de inputs intermedios que hace a estas ramas. Seguidamente se encuentra la ganadería que presenta ligazones relevantes con ramas pertenecientes al CAA. En tercer lugar aparece la rama de caza, silvicultura y pesca, poco relevante en el conjunto del CAA (véase Cuadro 2) pero que presenta ligazones relevantes con ramas de actividad ajenas al CAA.

En cuanto a la IAA, en primer lugar se sitúan las ramas de industrias de productos cárnicos y otras industrias de alimentación. Una rama que no pertenece al CAA pero que tiene ligazones relevantes con ramas de la IAA es la hostelería, destino importante de la producción elaborada por la industria transformadora de productos alimentarios.

Respecto a los cambios que se han producido en los quince años considerados, cabe destacar el aumento del coeficiente que presenta la agricultura con la industria vinícola, la fabricación de conservas vegetales y la construcción de maquinaria. Sorprende la reducción del coeficiente con la industria química. Analizando las cuatro componentes del coeficiente, se observa que la reducción se ha debido fundamentalmente a la disminución de la ligazón de oferta entre la industria química y la agricultura, es decir, a la disminución de la proporción de producción intermedia que se destina a la agricultura. 
Cuadro 3. Coeficientes de Streit del CAA de Navarra. 1980-1995.

\begin{tabular}{|c|c|c|c|c|c|}
\hline & & \multicolumn{2}{|c|}{ Interior } & \multicolumn{2}{|c|}{ Total } \\
\hline \multicolumn{2}{|c|}{ Ramas } & 1980 & 1995 & 1980 & 1995 \\
\hline 1. Agricultura & 1. Agricultura & 0,26 & 0,12 & 0,14 & 0,09 \\
\hline 1. Agricultura & 2. Ganadería & 0,17 & 0,14 & 0,14 & 0,12 \\
\hline 1. Agricultura & 3. Caza, silvicultura y pesca & 0,14 & 0,00 & 0,09 & 0,00 \\
\hline 1. Agricultura & 6. Fabric. de conservas vegetales & 0,24 & 0,29 & 0,19 & 0,26 \\
\hline 1. Agricultura & 9. Otras industrias de alimentación & 0,30 & 0,22 & 0,22 & 0,23 \\
\hline 1. Agricultura & 10. Industria vinícola & 0,03 & 0,15 & 0,02 & 0,13 \\
\hline 1. Agricultura & 19. Industria química & 0,15 & 0,06 & 0,14 & 0,10 \\
\hline 1. Agricultura & 28. Construcción de maquinaria & 0,03 & 0,15 & 0,01 & 0,06 \\
\hline 2. Ganadería & 2. Ganadería & 0,10 & 0,00 & 0,05 & 0,00 \\
\hline 2. Ganadería & 3. Caza, silvicultura y pesca & 0,10 & 0,14 & 0,05 & 0,05 \\
\hline 2. Ganadería & 5. Industria de productos cárnicos & 0,31 & 0,19 & 0,25 & 0,21 \\
\hline 2. Ganadería & 7. Industria de productos lácteos & 0,21 & 0,27 & 0,25 & 0,26 \\
\hline 2. Ganadería & 9. Otras industrias de alimentación & 0,26 & 0,35 & 0,26 & 0,30 \\
\hline 2. Ganadería & 49. Actividades sanitarias & 0,25 & 0,02 & 0,25 & 0,01 \\
\hline 3. Caza, silvicultura y pesca & 15. Industria de la madera & 0,17 & 0,06 & 0,17 & 0,10 \\
\hline 3. Caza, silvicultura y pesca & 16. Industria del papel & 0,18 & 0,10 & 0,16 & 0,11 \\
\hline 3. Caza, silvicultura y pesca & 28. Construcción de maquinaria & 0,02 & 0,15 & 0,02 & 0,13 \\
\hline 5. Industria productos cárnicos & 5. Industria de productos cárnicos & 0,46 & 0,45 & 0,49 & 0,41 \\
\hline 5. Industria productos cárnicos & 14. Industria del cuero y calzado & 0,09 & 0,01 & 0,11 & 0,03 \\
\hline 5. Industria productos cárnicos & 38. Hostelería & 0,09 & 0,11 & 0,06 & 0,09 \\
\hline 6. Fabric. de conservas vegetales & 6. Fabric. de conservas vegetales & 0,00 & 0,32 & 0,08 & 0,47 \\
\hline 6. Fabric. de conservas vegetales & 11. Otras industrias de bebidas & 0,00 & 0,00 & 0,16 & 0,00 \\
\hline 6. Fabric. de conservas vegetales & 38. Hostelería & 0,16 & 0,08 & 0,03 & 0,04 \\
\hline 7. Industria de productos lácteos & 7. Industria de productos lácteos & 0,12 & 0,01 & 0,18 & 0,15 \\
\hline 7. Industria de productos lácteos & 38. Hostelería & 0,14 & 0,10 & 0,07 & 0,12 \\
\hline 8. Pan, bollería y galletas & 9. Otras industrias de alimentación & 0,18 & 0,20 & 0,14 & 0,17 \\
\hline 8. Pan, bollería y galletas & 38. Hostelería & 0,25 & 0,23 & 0,23 & 0,22 \\
\hline 9. Otras industrias de alimentación & 9. Otras industrias de alimentación & 0,12 & 0,01 & 0,34 & 0,02 \\
\hline 10. Industria vinícola & 10. Industria vinícola & 0,48 & 0,66 & 0,42 & 0,54 \\
\hline 10. Industria vinícola & 11. Otras industrias de bebidas & 0,13 & 0,00 & 0,09 & 0,03 \\
\hline 11. Otras industrias de bebidas & 11. Otras industrias de bebidas & 0,02 & 0,16 & 0,09 & 0,39 \\
\hline 11. Otras industrias de bebidas & 38. Hostelería & 0,23 & 0,21 & 0,29 & 0,19 \\
\hline
\end{tabular}
Fuente: TION-80 y TION-95.

La ganadería ${ }^{10}$, aunque sigue teniendo relaciones relevantes con la industria de productos cárnicos, presenta una disminución importante del coeficiente, sobre todo en el interior de la región. Sin embargo, ha aumentado el grado de integración con la industria de productos lácteos y con las otras industrias de alimentación. En este caso, ese aumento también se produce en mayor medida en el interior de la región.

$10 \mathrm{El}$ elevado coeficiente que presenta con las actividades sanitarias en 1980 es un ejemplo de las limitaciones de estos coeficientes. En dicho año, las actividades sanitarias destinaron un $0,002 \%$ de su producción a usos intermedios. Esta producción se utilizó en un $99 \%$ en 
También es relevante el cambio producido en las relaciones que se establecen entre la hostelería y las IAA. En este caso, se debe analizar cómo ha variado la ligazón de oferta de las IAA con la hostelería y la ligazón de demanda de la hostelería con las IAA.

Así, el aumento del coeficiente con la industria de productos cárnicos se ha debido a que esta última rama destina mayor proporción de su producción intermedia a la hostelería.

Con la industria láctea la variación ha sido distinta según se considere el coeficiente interior o el total. La disminución del coeficiente interior se explica por la reducción de las dos ligazones señaladas, es decir, la hostelería demanda menor proporción de inputs intermedios a la industria láctea, y esta destina menor proporción de outputs intermedios a la hostelería. Sin embargo, el coeficiente total ha aumentado debido a la mayor proporción de productos lácteos que se utilizan en la hostelería. Por lo tanto, los consumos intermedios de esta rama han variado en el sentido de una mayor participación, en términos relativos, de los productos lácteos importados.

La variación del coeficiente con las industrias de conservas vegetales se explica básicamente por la disminución en la proporción de producción interior de esta rama que se destina a la hostelería.

Además se han producido cambios en las relaciones que cada rama establece consigo misma, siendo los más significativos los aumentos presentes en la fabricación de conservas vegetales y las otras industrias de bebidas, y la disminución en las otras industrias de alimentación.

Estos coeficientes nos permiten comprender las relaciones que se establecen entre cada par de ramas. Streit también propuso un coeficiente que mide las relaciones que una rama determinada mantiene con el conjunto del sistema productivo, denominado coeficiente de ligazón global (CLG) que expresa la intensidad de las relaciones de oferta y demanda que una rama mantiene con todas las demás. Este índice ${ }^{11}$ se calcula para cada rama como la suma de todos los coeficientes simétricos de Streit de dicha rama. Por lo tanto se expresa como $\mathrm{CLG}_{\mathrm{j}}=\sum_{\mathrm{i}} \mathrm{CES}_{\mathrm{ij}}$.

la ganadería, por lo que una ligazón de oferta era casi la unidad. Por lo tanto, aunque la relación entre las ramas es insignificante en términos absolutos, el coeficiente de Streit obtenido es muy elevado.

11 Titos (1974) señala que estos coeficientes son más satisfactorios puesto que en una sola cifra agrupan la medida global de las relaciones de oferta y de demanda existentes entre dos ramas cualesquiera. Pero también presentan limitaciones, siendo las más relevantes que estos coeficientes priman a las ramas que concentran la oferta y la demanda por insignificantes que sean en términos absolutos y que se tratan igual las relaciones hacia atrás que las relaciones hacia adelante (Cuadrado y Mancha, 1984). 
Los coeficientes de ligazón global para las ramas del CAA vienen recogidos en el Cuadro 4. El umbral de relevancia que se suele utilizar para valorar estos coeficientes es el valor del coeficiente de ligazón global medio del conjunto de ramas consideradas. En nuestro caso dicho umbral sería 0,951 para el coeficiente interior y 0,937 para el total en 1995. En el caso de 1980, dichos umbrales serían 0,920 y 0,887 respectivamente.

El primer resultado que se observa es que el conjunto de ramas de la economía navarra, como media, ha aumentado el valor de este coeficiente, tanto interior como total. Sin embargo, la media de las ramas del CAA lo han disminuido, por lo que en principio parece que su grado de articulación está disminuyendo, con la excepción de la rama de fabricación de conservas vegetales, que presenta un sensible aumento de su coeficiente de ligazón global tanto interior como total.

Cuadro 4. Coeficientes de ligazón global

\begin{tabular}{lcccccc}
\hline Rama & \multicolumn{3}{c}{ CLG Interior } & \multicolumn{3}{c}{ CLG Total } \\
\hline & 1980 & 1995 & DIF (2) & 1980 & 1995 & DIF (2) \\
\hline 1. Agricultura & 1,55 & 1,32 & $-0,23$ & 1,18 & 1,19 & 0,01 \\
2. Ganadería & 1,51 & 1,21 & $-0,30$ & 1,36 & 1,06 & $-0,30$ \\
3. Caza, silvicultura y pesca & 0,78 & 0,59 & $-0,19$ & 0,68 & 0,64 & $-0,04$ \\
Agricultura, ganadería y silvicultura (1) & $\mathbf{1 , 2 8}$ & $\mathbf{1 , 0 4}$ & $\mathbf{- 0 , 2 4}$ & $\mathbf{1 , 0 7}$ & $\mathbf{0 , 9 7}$ & $\mathbf{- 0 , 1 0}$ \\
5. Industria de productos cárnicos & 1,12 & 0,94 & $-0,18$ & 1,04 & 1,02 & $-0,02$ \\
6. Fabricación de conservas vegetales & 0,71 & 0,89 & 0,18 & 0,77 & 0,95 & 0,18 \\
7. Industria de productos lácteos & 0,75 & 0,63 & $-0,12$ & 0,81 & 0,71 & $-0,10$ \\
8. Pan, bollería y galletas & 0,65 & 0,69 & 0,04 & 0,64 & 0,61 & $-0,03$ \\
9. Otras industrias de alimentación & 1,16 & 0,97 & $-0,19$ & 1,36 & 1,03 & $-0,33$ \\
10. Industria vinícola & 0,87 & 0,90 & 0,03 & 0,89 & 0,85 & $-0,04$ \\
11. Otras industrias de bebida y tabaco & 0,68 & 0,65 & $-0,03$ & 0,91 & 0,88 & $-0,03$ \\
Industria Agroalimentaria (1) & $\mathbf{0 , 8 5}$ & $\mathbf{0 , 8 1}$ & $\mathbf{- 0 , 0 4}$ & $\mathbf{0 , 9 2}$ & $\mathbf{0 , 8 6}$ & $\mathbf{- 0 , 0 6}$ \\
Complejo Agroalimentario (1) & $\mathbf{0 , 9 8}$ & $\mathbf{0 , 8 8}$ & $\mathbf{- 0 , 1 0}$ & $\mathbf{0 , 9 7}$ & $\mathbf{0 , 8 9}$ & $\mathbf{- 0 , 0 8}$ \\
Economía Navarra (1) & 0,92 & 0,97 & 0,05 & 0,89 & 0,98 & 0,09 \\
\hline F & & &
\end{tabular}

Fuente: TION-80 y TION-95. (1) Los valores presentados son las medias de las ramas incluidas en cada agrupación. (2) Esta diferencia se calcula como el valor en 1995 menos el valor en 1980.

Con los umbrales de relevancia señalados y analizando los coeficientes interiores, del conjunto de ramas que se incluyen en el CAA las que tienen ligazones relevantes en los dos años considerados son la agricultura y la ganadería, seguidas por otras industrias de alimentación y las industrias de productos cárnicos. Sin embargo, también son estas ramas las que han visto diminuir sensiblemente sus coeficientes de ligazón global en el período de análisis. 


\subsection{Coeficientes de Chenery y Watanabe}

Otra forma de cuantificar el impacto directo de una rama sobre el resto es a través de los coeficientes de Chenery y Watanabe (1958), quienes efectuaron una cuantificación de los encadenamientos, seleccionando aquellas actividades cuyos efectos eran superiores a la media mediante la combinación de dos criterios.

Los eslabonamientos hacia atrás para cada rama los miden a través del coeficiente $\mu_{\mathrm{j}}=\sum_{\mathrm{i}} \mathrm{x}_{\mathrm{ij}} / \mathrm{X}_{\mathrm{j}}$, es decir, la proporción de los inputs intermedios respecto a la producción de la rama $j$.

Los eslabonamientos hacia adelante para cada rama los cuantifican a través del coeficiente $\omega_{\mathrm{i}}=\sum_{\mathrm{j}} \mathrm{x}_{\mathrm{ij}} / \mathrm{Z}_{\mathrm{i}}$, o proporción del output de la rama $i$ que se destina a utilizaciones intermedias respecto al total de destinos $\left(\mathrm{Z}_{\mathrm{i}}\right)$ de la misma.

Los coeficientes obtenidos ${ }^{12}$, interiores y totales, para las ramas pertenecientes al CAA se recogen en el Cuadro 5. Las ramas las que presentan mayor capacidad de arrastre en el interior de la región son la ganadería, la industria de fabricación de conservas vegetales y la industria vinícola, y además son las que han aumentado en mayor medida esta capacidad en el período considerado.

Dado que la capacidad de arrastre total apenas ha variado, se puede señalar que en estos quince años se ha registrado un proceso de sustitución de inputs intermedios importados por inputs intermedios procedentes de la región. En este sentido también destaca la rama de pan, bollería y galletas, ya que aumenta sensiblemente su capacidad de arrastre interior y disminuye la total. Sin embargo, se ha producido una sustitución inversa en la rama de productos cárnicos, ya que era la que presentaba en 1980 mayor capacidad de empuje en el interior de la región, y la que ha soportado una mayor disminución en este coeficiente.

Respecto a la capacidad de empuje, destacan la rama de agricultura por su importante papel como oferente de inputs intermedios a la IAA, y la rama de otras industrias de alimentación, donde se incluyen los fabricantes de harina y piensos para la alimentación animal, productos utilizados por las ramas de pan, bollería y galletas y la ganadería, respectivamente, para elaborar su producción. Sin embargo, el comportamiento de ambas ha

\footnotetext{
12 Muñoz Cidad (1994) señala que la utilidad de estos coeficientes es hasta cierto punto limitada ya que se utilizan coeficientes directos, no totales, lo cuales podrían suministrar información más completa. Además son coeficientes medios, sin ponderaciones, por lo que no deben extraerse conclusiones precipitadas, ya que una rama con coeficientes superiores a la media puede ser económicamente irrelevante. Por
} 
sido dispar. Así, la agricultura ha aumentado su capacidad de empuje interior y total, mientras que las otras industrias de alimentación solamente han aumentado su capacidad de empuje interior, de lo cual se deduce que han aumentado sus ventas intermedias en la región.

Cuadro 5. Coeficientes de Chenery y Watanabe.

\begin{tabular}{|c|c|c|c|c|c|c|c|c|c|c|c|c|}
\hline & \multicolumn{6}{|c|}{ ATRÁS } & \multicolumn{6}{|c|}{ ADELANTE } \\
\hline & \multicolumn{3}{|c|}{ Interior } & \multicolumn{3}{|c|}{ Total } & \multicolumn{3}{|c|}{ Interior } & \multicolumn{3}{|c|}{ Total } \\
\hline & 1980 & 1995 & DIF & 1980 & 1995 & DIF & 1980 & 1995 & DIF & 1980 & 1995 & DIF \\
\hline 1. Agricultura & 0,16 & 0,26 & 0,09 & 0,35 & 0,45 & 0,09 & 0,39 & 0,85 & 0,46 & 0,45 & 0,78 & 0,33 \\
\hline 2. Ganadería & 0,26 & 0,53 & 0,27 & 0,74 & 0,72 & $-0,02$ & 0,51 & 0,28 & $-0,23$ & 0,58 & 0,37 & $-0,22$ \\
\hline 3. Caza, silvicultura y pesca & 0,11 & 0,01 & $-0,10$ & 0,17 & 0,01 & $-0,16$ & 0,76 & 0,85 & 0,09 & 0,70 & 0,63 & $-0,07$ \\
\hline Agricultura, ganadería y silvic. (1) & 0,18 & 0,26 & 0,09 & 0,42 & $\mathbf{0 , 3 9}$ & $-0,03$ & $\mathbf{0 , 5 5}$ & 0,66 & $\mathbf{0 , 1 1}$ & $\mathbf{0 , 5 8}$ & $\mathbf{0 , 5 9}$ & $\mathbf{0 , 0 1}$ \\
\hline 5. Industria de productos cárnicos & 0,56 & 0,43 & $-0,14$ & 0,81 & 0,79 & $-0,02$ & 0,18 & 0,23 & 0,05 & 0,23 & 0,29 & 0,06 \\
\hline 6. Fabricación conservas vegetales & 0,37 & 0,50 & 0,13 & 0,72 & 0,78 & 0,06 & 0,00 & 0,02 & 0,02 & 0,01 & 0,08 & 0,07 \\
\hline 7. Industria de productos lácteos & 0,29 & 0,44 & 0,15 & 0,82 & 0,82 & 0,00 & 0,04 & 0,04 & 0,00 & 0,08 & 0,08 & $-0,01$ \\
\hline 8. Pan, bollería y galletas & 0,21 & 0,39 & 0,18 & 0,69 & 0,55 & $-0,14$ & 0,04 & 0,09 & 0,05 & 0,03 & 0,09 & 0,06 \\
\hline 9. Otras industrias de alimentación & 0,31 & 0,38 & 0,07 & 0,80 & 0,81 & 0,01 & 0,17 & 0,45 & 0,28 & 0,40 & 0,43 & 0,03 \\
\hline 10. Industria vinícola & 0,23 & 0,53 & 0,30 & 0,64 & 0,65 & 0,01 & 0,14 & 0,19 & 0,05 & 0,25 & 0,21 & $-0,04$ \\
\hline 11. Otras industrias de bebidas y tab. & 0,15 & 0,120 & 0,04 & 0,64 & 0,71 & 0,07 & 0,15 & 0,06 & $-0,09$ & 0,34 & 0,28 & $-0,06$ \\
\hline Industria Agroalimentaria (1) & $\mathbf{0 , 3 0}$ & 0,41 & $\mathbf{0 , 1 1}$ & $\mathbf{0 , 7 3}$ & $\mathbf{0 , 7 3}$ & $\mathbf{0 , 0 0}$ & $\mathbf{0 , 1 0}$ & 0,15 & $\mathbf{0 , 0 5}$ & $\mathbf{0 , 1 9}$ & 0,21 & $\mathbf{0 , 0 2}$ \\
\hline Complejo Agroalimentario (1) & 0,27 & $\mathbf{0 , 3 7}$ & $\mathbf{0 , 1 0}$ & 0,64 & $\mathbf{0 , 6 3}$ & $-0,01$ & 0,24 & $\mathbf{0 , 3 0}$ & $\mathbf{0 , 0 7}$ & $\mathbf{0 , 3 1}$ & $\mathbf{0 , 3 2}$ & $\mathbf{0 , 0 2}$ \\
\hline Economía Navarra (1) & 0,19 & 0,26 & 0,07 & 0,52 & 0,54 & 0,02 & 0,25 & 0,29 & 0,04 & 0,37 & 0,39 & 0,02 \\
\hline
\end{tabular}

En este caso destaca nuevamente la de ganadería, rama que partiendo en 1980 con la mayor capacidad de empuje, tiene en el período considerado la mayor disminución.

La combinación de ambos coeficientes permite clasificar las ramas de una TIO de acuerdo con los dos efectos. En el Cuadro 6 se presenta dicha clasificación para las ramas del CAA de Navarra, en 1980 y 1995.

En la primera línea (I) se encuentran las ramas que demandan muchos inputs y a la vez son oferentes de outputs intermedios. Si hacemos referencia a la clasificación en 1995, de esta forma se clasificaría la rama de otras industrias de alimentación, donde se incluyen fundamentalmente actividades elaboradoras de productos que se utilizan como inputs intermedios en otras ramas

En la segunda línea (II) se incluyen las ramas son importantes demandantes de inputs para realizar su proceso productivo, pero su producción se destina de forma superior a la media a la demanda final. Lógicamente, aquí se incluyen la mayoría de las ramas

último, son coeficientes que no tienen en cuenta si las relaciones de una rama con el resto están muy concentradas, de modo que dos ramas con los mismos coeficientes pueden tener una incidencia económica diferente en función de la concentración. 
pertenecientes a la IAA, cinco de las siete consideradas. La rama ganadería también se incluye en esta línea.

En la tercera (III) se sitúan aquellas ramas cuya producción tiene mayoritariamente un destino intermedio pero que a su vez utilizan pocos inputs intermedios. En nuestro caso aparecen la rama agricultura y la de caza, silvicultura y pesca.

Si analizamos los cambios que se han producido respecto a la situación en 1980, se observa cómo las otras industrias de alimentación han pasado a tener una capacidad regional de empuje superior a la media, lo cual nos indica que la producción intermedia de esta rama tiene un destino regional. Por otra parte, la ganadería pasa a clasificarse como una rama con fuertes vínculos hacia atrás pero cuya producción se destina de forma superior a la media de la economía a otros usos distintos de los intermedios.

Cuadro 6. Clasificación de las ramas productivas del CAA de acuerdo con los coeficientes de Chenery y Watanabe (1).

\begin{tabular}{|c|c|c|}
\hline & 1980 & 1995 \\
\hline I. $\mu_{j}>\bar{\mu}_{j}, \omega_{i}>\bar{\omega}_{i}$ & $\begin{array}{l}\text { 2. Ganadería } \\
\text { 2. Ganadería } \\
\text { 9. Otras industrias de alimentación }\end{array}$ & $\begin{array}{l}\text { 9. Otras industrias de alimentación } \\
\text { 9. Otras industrias de alimentación }\end{array}$ \\
\hline II. $\mu_{j}>\bar{\mu}_{j}, \omega_{i}<\bar{\omega}_{i}$ & $\begin{array}{l}\text { 5. Industria de productos cárnicos } \\
\text { 6. Fabricación de conservas vegetales } \\
\text { 7. Industria de productos lácteos } \\
\text { 8. Pan, bollería y galletas } \\
\text { 9. Otras industrias de alimentación } \\
\text { 10. Industria vinícola } \\
\text { 5. Industria de productos cárnicos } \\
\text { 6. Fabricación de conservas vegetales } \\
\text { 7. Industria de productos lácteos } \\
\text { 8. Pan, bollería y galletas } \\
\text { 10. Industria vinícola } \\
\text { 11. Otras industrias de bebida y tabaco }\end{array}$ & $\begin{array}{l}\text { 2. Ganadería } \\
\text { 5. Industria de productos cárnicos } \\
\text { 6. Fabricación de conservas vegetales } \\
\text { 7. Industria de productos lácteos } \\
\text { 8. Pan, bollería y galletas } \\
\\
\text { 10. Industria vinícola } \\
\text { 2. Ganadería } \\
\text { 5. Industria de productos cárnicos } \\
\text { 6. Fabricación de conservas vegetales } \\
\text { 7. Industria de productos lácteos } \\
\text { 8. Pan, bollería y galletas } \\
\text { 10. Industria vinícola } \\
\text { 11. Otras industrias de bebida y tabaco }\end{array}$ \\
\hline III. $\mu_{j}<\bar{\mu}_{j}, \omega_{i}>\bar{\omega}_{i}$ & $\begin{array}{l}\text { 1. Agricultura } \\
\text { 3. Caza, silvicultura y pesca } \\
\text { 1. Agricultura } \\
\text { 3. Caza, silvicultura y pesca }\end{array}$ & $\begin{array}{l}\text { 1. Agricultura } \\
\text { 3. Caza, silvicultura y pesca } \\
\text { 1. Agricultura } \\
\text { 3. Caza, silvicultura y pesca }\end{array}$ \\
\hline IV. $\mu_{\mathrm{j}}<\bar{\mu}_{\mathrm{j}}, \omega_{\mathrm{i}}<\bar{\omega}_{\mathrm{i}}$ & 11. Otras industrias de bebida y tabaco & 11. Otras industrias de bebida y tabaco \\
\hline
\end{tabular}

Fuente: TION80 y TION95. (1) La clasificación de las ramas se ha realizado comparando los valores con la media de las 51 ramas de actividad. En cursiva aparece la clasificación de acuerdo a los coeficientes totales.

Por último, se encuentran las ramas de actividad con escasa capacidad para crear vínculos hacia adelante y hacia atrás, que se sitúan en la cuarta línea (IV). En esta situación se encuentra la rama de otras industrias de bebidas y tabaco, por lo que su producción tiene un 
uso fundamentalmente final, y requiere pocos inputs intermedios regionales para su producción.

Si se consideran los flujos totales, solamente la rama de otras industrias de bebidas y tabaco cambiaría su clasificación. Así, esta rama pasaría a presentar una capacidad de arrastre superior a la media, lo cual implica que una parte importante de sus consumos intermedios proceden de fuera de Navarra.

\subsection{Efectos difusión y absorción y coeficientes de Rasmussen.}

Como se ha señalado anteriormente, los coeficientes utilizados hasta ahora ofrecen una visión parcial de las interdependencias sectoriales, ya que no tienen en cuenta en la cuantificación de los eslabonamientos los efectos indirectos. Con el fin de considerarlos, se hace uso de la inversa de la matriz de Leontief. Cada elemento de dicha matriz, $r_{\mathrm{ij}}$, expresa la cantidad de producto que directa e indirectamente se necesita de la rama $i$ para que la rama $j$ pueda suministrar una unidad de producto a la demanda final.

En base a esta matriz, se pueden elaborar distintos indicadores. El efecto difusión o multiplicador de la producción se obtiene sumando cada una de las columnas de la matriz inversa de Leontief, e indica el impacto que tendrá sobre todas las ramas productivas un aumento de una unidad en la demanda final de la rama correspondiente. Por lo tanto recoge los efectos hacia atrás, nos da una aproximación de la intensidad con la que un sector difunde en el sistema económico una variación unitaria de su demanda final. Este indicador se expresa como $\mathrm{ED}_{\mathrm{j}}=\sum_{\mathrm{i}} \mathrm{r}_{\mathrm{ij}}=\mathrm{r}_{\mathrm{j}}$.

El efecto absorción o valor de la expansión uniforme de la demanda: se obtiene sumando cada una de las filas de la matriz inversa de Leontief, e indica cómo variará la producción de un sector cuando varía de forma unitaria la demanda final de todas las ramas de la economía. Este indicador recoge los efectos hacia adelante ya que expresa la intensidad con la que la rama correspondiente absorbe las variaciones de la demanda final de todas las ramas de actividad y se expresa como $\mathrm{EA}_{\mathrm{i}}=\sum_{\mathrm{j}} \mathrm{r}_{\mathrm{ij}}=\mathrm{r}_{\mathrm{i} \text {. }}$.

En el Cuadro 7 se recogen los efectos difusión y absorción interiores y totales para las ramas productivas pertenecientes al CAA.

Si se analiza el efecto difusión, la industria vinícola, la industria de productos lácteos y la ganadería presentan los mayores valores, y por lo tanto mayores capacidades de arrastre. Las tres ramas señaladas han aumentado de forma considerable en los quince años 
considerados el efecto que producen en el resto del sistema económico. En 1980 era la rama de productos cárnicos la que presentaba un mayor efecto difusión, y aunque en 1995 sigue siendo una rama relativamente importante en este sentido, es la única de las pertenecientes al CAA que ha experimentado en el período una disminución de esta capacidad.

Respecto al efecto absorción o capacidad de empuje, la agricultura, las otras industrias de alimentación y la ganadería presentan los valores más elevados, y por lo tanto, deben aumentar su producción en mayor cuantía ante un incremento unitario en la demanda final de todas las ramas de la TIO. Aunque esta clasificación no ha variado en el período considerado, el comportamiento de las tres ramas señaladas ha sido diferente. La primera, la agricultura, ha aumentado su capacidad de empuje interior y total. Las otras industrias de alimentación han aumentado su capacidad interior y han disminuido la total. La ganadería ha disminuido ambas. Estos resultados corroboran los obtenidos a partir de los coeficientes directos.

Cuadro 7. Efectos difusión y absorción.

\begin{tabular}{|c|c|c|c|c|c|c|c|c|c|c|c|c|}
\hline & \multicolumn{6}{|c|}{ DIFUSIÓN } & \multicolumn{6}{|c|}{ ABSORCIÓN } \\
\hline & \multicolumn{3}{|c|}{ Interior } & \multicolumn{3}{|c|}{ Total } & \multicolumn{3}{|c|}{ Interior } & \multicolumn{3}{|c|}{ Total } \\
\hline & 1980 & 1995 & DIF & 1980 & 1995 & DIF & 1980 & 1995 & DIF & 1980 & 1995 & DIF \\
\hline 1. Agricultura & 1,20 & 1,37 & 0,17 & 1,73 & 2,02 & 0,29 & 1,97 & 2,35 & 0,38 & 3,61 & 3,87 & 0,26 \\
\hline 2. Ganadería & 1,33 & 1,76 & 0,43 & 2,73 & 2,71 & $-0,02$ & 1,88 & 1,64 & $-0,24$ & 2,66 & 2,45 & $-0,21$ \\
\hline 3. Caza, silvicultura y pesca & 0,14 & 1,01 & 0,87 & 1,34 & 1,03 & $-0,31$ & 1,21 & 1,15 & $-0,06$ & 1,95 & 1,68 & $-0,27$ \\
\hline Agricultura, ganadería y silv. (1) & $\mathbf{0 , 8 9}$ & 1,38 & 0,49 & 1,93 & 1,92 & $-0,01$ & 1,69 & 1,71 & 0,02 & 2,74 & 2,67 & $-0,07$ \\
\hline 5. Industria de productos cárnicos & 1,80 & 1,69 & $-0,11$ & 3,26 & 3,06 & $-0,20$ & 1,27 & 1,25 & $-0,02$ & 1,73 & 2,16 & 0,43 \\
\hline 6. Fabricación conservas vegetales & 1,45 & 1,69 & 0,24 & 2,45 & 2,59 & 0,14 & 1,00 & 1,02 & 0,02 & 1,04 & 1,10 & 0,06 \\
\hline 7. Industria de productos lácteos & 1,38 & 1,72 & 0,34 & 3,08 & 3,08 & $-0,00$ & 1,04 & 1,02 & $-0,02$ & 1,12 & 1,09 & $-0,03$ \\
\hline 8. Pan, bollería y galletas & 1,28 & 1,57 & 0,29 & 2,66 & 2,33 & $-0,33$ & 1,02 & 1,03 & 0,01 & 1,02 & 1,05 & 0,03 \\
\hline 9. Otras industrias de alimentación & 1,37 & 1,52 & 0,15 & 2,70 & 2,73 & 0,03 & 1,45 & 1,78 & 0,33 & 3,88 & 2,55 & $-1,33$ \\
\hline 10. Industria vinícola & 1,29 & 1,80 & 0,51 & 2,28 & 2,35 & 0,07 & 1,14 & 1,23 & 0,09 & 1,45 & 1,28 & $-0,17$ \\
\hline 11. Otras industrias de bebidas y tab. & 1,19 & 1,26 & 0,07 & 2,43 & 2,63 & 0,20 & 1,06 & 1,04 & $-0,02$ & 1,34 & 1,58 & 0,24 \\
\hline Industria Agroalimentaria (1) & 1,39 & 1,61 & $\mathbf{0 , 2 2}$ & 2,69 & 2,68 & $-0,01$ & 1,14 & 1,20 & 0,06 & 1,65 & $\mathbf{1 , 5 4}$ & $-0,11$ \\
\hline Complejo Agroalimentario (1) & 1,24 & 1,54 & $\mathbf{0 , 3 0}$ & 2,47 & 2,45 & $-0,02$ & 1,30 & 1,35 & 0,05 & 1,98 & $\mathbf{1 , 8 8}$ & $-0,10$ \\
\hline Economía Navarra (1) & 1,23 & 1,37 & 0,14 & 2,23 & 2,26 & 0,03 & 1,23 & 1,37 & 0,14 & 2,23 & 2,26 & 0,03 \\
\hline
\end{tabular}

Fuente: TION-80 y TION-95. (1) Los valores presentados son las medias de las ramas incluidas en cada agrupación.

Reformulando los efectos difusión y absorción, se obtienen los coeficientes de Rasmussen (1956). El primero de ellos, el denominado poder de dispersión de los efectos de una rama sobre el resto del sistema, se expresa como $U_{. j}=\frac{r_{. j} / n}{(1 / n) \sum_{j}\left(r_{. j} / n\right)}$, donde $n$ es el número de ramas y $r_{. j}$ es el efecto difusión de cada rama. Se trata de una medida relativa de la fuerza con que un incremento en la demanda $j$ se dispersa a través del sistema económico. $\mathrm{Si}$ $\mathrm{U}_{. \mathrm{j}}>1$, significa que la rama $j$ tiene una capacidad de arrastre superior a la media. 
El coeficiente de sensibilidad de la dispersión trata de medir cómo un incremento en la demanda de todo el sistema "tira" de la rama $i$, y se define como $\mathrm{U}_{\mathrm{i} .}=\frac{\mathrm{r}_{\mathrm{i} .} / \mathrm{n}}{(1 / \mathrm{n}) \sum_{\mathrm{i}}\left(\mathrm{r}_{\mathrm{i} .} / \mathrm{n}\right)}$, siendo $r_{i}$ el efecto absorción de cada rama. Cuando dicho coeficiente es mayor que la unidad significa que la rama en cuestión es arrastrada de forma superior a la media.

Para Domingo et al. (1987) la ventaja de los índices de Rasmussen estriba en que permite realizar comparaciones interindustriales sobre la base de que se normaliza los promedios parciales, relacionándolos con el promedio total. Ahora bien, como señala Muñoz Cidad (1988), es importante incorporar a dichos coeficientes un elemento que tenga en cuenta la importancia de cada rama en la demanda final, así como su grado de concentración en los encadenamientos. Para atender ambas cuestiones pueden perfeccionarse estos índices de la siguiente manera.

En relación con el primer aspecto se pueden incluir ponderaciones, de forma que se destacaría mejor el poder de dispersión o la sensibilidad de las diferentes ramas. En nuestro caso, se han ponderado los efectos en función de la participación de cada rama en la demanda final.

Para valorar la distinta concentración de los efectos se pueden elaborar coeficientes de variación. Cuanto mayores sean estos, más concentrados se encuentran los efectos de la rama correspondiente, y cuanto menores sean, más homogéneamente distribuidos se encuentran los efectos entre las actividades productivas que integran el sistema.

Teniendo en cuenta los criterios precedentes, Titos (1995) sugiere la siguiente clasificación sobre el comportamiento estratégico de las ramas productivas:

- Ramas impulsoras: aquellas que ostentan un coeficiente de poder de dispersión superior a la unidad con un coeficiente de variación inferior a la media. Son ramas que cuando aumenta su demanda final posibilitan que el conjunto de producciones sectoriales se incremente considerablemente.

- Ramas receptoras: aquellas que presentan un coeficiente de sensibilidad de la dispersión superior a la unidad con un coeficiente de variación inferior a la media. Son ramas cuya producción debe aumentar bastante cuando se producen incrementos generalizados en las demandas finales, son receptoras de efectos multiplicadores de demanda. Si no respondieran bien a estos efectos, ocasionarían estrangulamientos en el sistema productivo.

- Ramas clave: aquellas que se comportan a la vez como impulsoras y receptoras, y que por tanto se muestran como doblemente estratégicas para el sistema productivo. 
En el Cuadro 8 aparecen los coeficientes de Rasmussen, ponderados por la participación de cada rama en la demanda final. En 1995 las ramas con mayor poder de dispersión regional son la ganadería y la fabricación de conservas vegetales, cuyos coeficientes han sido además los que más han aumentado en el período. Si consideramos los coeficientes totales, en esta situación también se encontrarían las industrias de productos cárnicos, a pesar de la disminución en su capacidad de arrastre tanto interior como total.

En cuanto al coeficiente de sensibilidad de la dispersión, destacan por sus mayores valores las ramas de fabricación de conservas vegetales junto con la agricultura, que por lo tanto son las que presentan mayor capacidad de empuje.

Es destacable la sensible disminución que se produce en las capacidades de empuje y arrastre para las ramas de agricultura y otras industrias de alimentación al incorporar las ponderaciones en la elaboración de los coeficientes.

Cuadro 8. Coeficientes de Rasmussen (1).

\begin{tabular}{|c|c|c|c|c|c|c|c|c|c|c|c|c|}
\hline & \multicolumn{6}{|c|}{ PODER $U_{. j}$} & \multicolumn{6}{|c|}{ SENSIBILIDAD U $\mathrm{U}_{\mathrm{i}}$} \\
\hline & \multicolumn{3}{|c|}{ Interior } & \multicolumn{3}{|c|}{ Total } & \multicolumn{3}{|c|}{ Interior } & \multicolumn{3}{|c|}{ Total } \\
\hline & 1980 & 1995 & DIF & 1980 & 1995 & DIF & 1980 & 1995 & DIF & 1980 & 1995 & DIF \\
\hline 1. Agricultura & 1,87 & 0,21 & $-1,66$ & 1,34 & 0,36 & $-0,98$ & 2,53 & 1,00 & $-1,53$ & 2,05 & 1,18 & $-0,87$ \\
\hline 2. Ganadería & 0,98 & 1,07 & 0,09 & 0,94 & 0,84 & $-0,10$ & 1,50 & 0,84 & $-0,66$ & 1,09 & 0,75 & $-0,34$ \\
\hline 3. Caza, silvicultura y pesca & 0,06 & 0,01 & $-0,05$ & 0,12 & 0,08 & $-0,04$ & 0,21 & 0,09 & $-0,12$ & 0,48 & 0,31 & $-0,17$ \\
\hline Agricultura, ganadería y silvic. (2) & $\mathbf{0 , 9 7}$ & $\mathbf{0 , 4 3}$ & $-0,54$ & $\mathbf{0 , 8 0}$ & $\mathbf{0 , 4 3}$ & $-0,37$ & 1,41 & 0,65 & $-0,76$ & 1,21 & 0,74 & $-0,47$ \\
\hline 5. Industria de productos cárnicos & 1,65 & 0,80 & $-0,85$ & 1,81 & 1,27 & $-0,54$ & 1,12 & 0,62 & $-0,50$ & 0,85 & 0,83 & $-0,02$ \\
\hline 6. Fabricación conservas vegetales & 1,31 & 2,01 & 0,70 & 0,99 & 1,49 & 0,50 & 0,91 & 1,21 & 0,30 & 0,41 & 0,63 & 0,22 \\
\hline 7. Industria de productos lácteos & 1,11 & 0,45 & $-0,66$ & 1,19 & 0,67 & $-0,52$ & 0,84 & 0,27 & $-0,57$ & 0,44 & 0,24 & $-0,20$ \\
\hline 8. Pan, bollería y galletas & 0,54 & 0,41 & $-0,13$ & 0,63 & 0,36 & $-0,27$ & 0,44 & 0,29 & $-0,15$ & 0,25 & 0,17 & $-0,08$ \\
\hline 9. Otras industrias de alimentación & 2,19 & 0,66 & $-1,53$ & 2,52 & 0,91 & $-1,61$ & 1,92 & 0,79 & $-1,13$ & 2,21 & 0,80 & $-1,41$ \\
\hline 10. Industria vinícola & 0,38 & 0,70 & 0,32 & 0,41 & 0,45 & 0,04 & 0,34 & 0,48 & 0,14 & 0,28 & 0,26 & $-0,02$ \\
\hline 11. Otras industrias de bebidas y tab. & 0,36 & 0,72 & 0,36 & 0,45 & 0,98 & 0,53 & 0,35 & 0,61 & 0,26 & 0,32 & 0,63 & 0,31 \\
\hline Industria Agroalimentaria (2) & 1,08 & $\mathbf{0 , 8 2}$ & $-0,26$ & 1,14 & 0,87 & $-0,27$ & $\mathbf{0 , 8 5}$ & 0,61 & $-0,24$ & 0,68 & 0,51 & $-0,17$ \\
\hline Complejo Agroalimentario (2) & 1,05 & $\mathbf{0 , 7 0}$ & $-0,35$ & 1,04 & 0,74 & $-0,30$ & 1,02 & 0,62 & $-0,40$ & $\mathbf{0 , 8 4}$ & $\mathbf{0 , 5 8}$ & $-0,26$ \\
\hline
\end{tabular}

La clasificación de las ramas de acuerdo a los coeficientes de Rasmussen interiores y a sus coeficientes de variación nos indica que como impulsoras se clasificarían en 1980 la fabricación de conservas vegetales, la industria de productos lácteos y las otras industrias de alimentación, mientras que en 1995 estarían en este grupo la primera y la ganadería. Como receptoras, en 1980 se situarían la agricultura y la ganadería y en 1990 solamente la primera. Por lo tanto, a partir de las dos TION consideradas no existiría en el CAA de Navarra ninguna rama que se pudiera clasificar como clave. 


\section{CAPACIDAD DE GENERACIÓN DE RENTA Y EMPLEO}

Con el fin de evaluar la capacidad de generar renta por parte de las ramas pertenecientes al CAA se utilizan el coeficiente directo y el multiplicador de renta simple.

El primero nos indica la proporción de valor añadido en la producción efectiva de cada rama, y se expresa como $\mathrm{v}_{\mathrm{i}}=\mathrm{VAB}_{\mathrm{i}} / \mathrm{PE}_{\mathrm{i}}$.

El segundo cuantifica la capacidad de generar valor añadido, directa e indirectamente en el sistema económico a través de un incremento de una unidad en la demanda final de cada rama. Su expresión sería y $\mathrm{MR}_{\mathrm{j}}=\sum_{\mathrm{i}} \mathrm{v}_{\mathrm{i}} \mathrm{r}_{\mathrm{ij}}$, donde $\mathrm{r}_{\mathrm{ij}}$ es el elemento correspondiente de la inversa de la matriz de Leontief interior.

Los coeficientes obtenidos para cada una de las ramas del CAA se pueden ver en el Cuadro 9. A la vista de los resultados se observa que en 1995 el CAA tiene, como media, una capacidad de generar renta directa inferior a la media del conjunto regional, aunque si se considera también la de generar renta indirectamente, su capacidad es algo superior a la media regional. Una circunstancia destacable es que en los quince años contemplados ha aumentado dicha capacidad en una cuantía sensiblemente superior a la media de las 51 ramas consideradas en las TION.

Cuadro 9. Capacidad de generación de renta.

\begin{tabular}{lcccccc}
\hline & & & & & $\mathrm{MR}_{\mathrm{j}}$ \\
\hline & 1980 & 1995 & $\mathrm{DIF}$ & 1980 & 1995 & $\mathrm{DIF}$ \\
\hline 1. Agricultura & 0,66 & 0,78 & 0,12 & 0,76 & 0,95 & 0,19 \\
2. Ganadería & 0,26 & 0,38 & 0,12 & 0,40 & 0,73 & 0,33 \\
3. Caza, silvicultura y pesca & 0,83 & 0,99 & 0,16 & 0,90 & 0,99 & 0,09 \\
Agricultura, ganadería y silvic. (1) & $\mathbf{0 , 5 8}$ & $\mathbf{0 , 7 2}$ & $\mathbf{0 , 1 4}$ & $\mathbf{0 , 6 9}$ & $\mathbf{0 , 8 9}$ & $\mathbf{0 , 2 0}$ \\
5. Industria de productos cárnicos & 0,18 & 0,21 & 0,03 & 0,41 & 0,50 & 0,09 \\
6. Fabricación de conservas vegetales & 0,28 & 0,25 & $-0,03$ & 0,53 & 0,70 & 0,17 \\
7. Industria de productos lácteos & 0,18 & 0,18 & 0,00 & 0,31 & 0,50 & 0,19 \\
8. Pan, bollería y galletas & 0,30 & 0,45 & 0,15 & 0,41 & 0,69 & 0,28 \\
9. Otras industrias de alimentación & 0,18 & 0,19 & 0,01 & 0,40 & 0,52 & 0,12 \\
10. Industria vinícola & 0,34 & 0,35 & 0,01 & 0,48 & 0,81 & 0,33 \\
11. Otras industrias de bebida y tabaco & 0,27 & 0,20 & $-0,07$ & 0,35 & 0,34 & $-0,01$ \\
Industria Agroalimentaria (1) & $\mathbf{0 , 2 5}$ & $\mathbf{0 , 2 6}$ & $\mathbf{0 , 0 1}$ & $\mathbf{0 , 4 1}$ & $\mathbf{0 , 5 8}$ & $\mathbf{0 , 1 7}$ \\
Complejo Agroalimentario (1) & $\mathbf{0 , 3 5}$ & $\mathbf{0 , 4 0}$ & $\mathbf{0 , 0 5}$ & $\mathbf{0 , 4 9}$ & $\mathbf{0 , 6 7}$ & $\mathbf{0 , 1 8}$ \\
Economía Navarra (1) & 0,45 & 0,46 & 0,01 & 0,56 & 0,65 & 0,09 \\
\hline Fuente: TION-80 y TION-95. (1) Los valores presentados son las medias de las ramas incluidas en cada agrupación, excepto en el caso del \\
multiplicador de empleo obtenido para el sector primario. & & & & & &
\end{tabular}


Las diferencias entre las ramas de actividad son importantes. Así, la rama agricultura es la que presenta una mayor capacidad, ya que por cada unidad de producción se generan 0,78 unidades de renta y por cada unidad de demanda final adicional se genera una renta en el territorio navarro de 0,95 unidades. Le siguen en orden de importancia la industria vinícola , la ganadería y las industrias de conservas vegetales y la de pan, bollería y galletas. Todas ellas presentaban también en 1980 las mayores capacidades, aunque destacan por un aumento muy apreciable de la capacidad total en los quince años considerados la ganadería y la industria vinícola.

Para calcular la capacidad de generar empleo utilizaremos los mismos indicadores. Así, se calcula el coeficiente directo de empleo $\mathrm{p}_{\mathrm{i}}$, que representa la relación entre las personas ocupadas en cada rama y la producción efectiva expresada en millones de pesetas. El multiplicador de empleo se obtiene como $\mathrm{MEMP}_{j}=\sum_{\mathrm{i}} \mathrm{p}_{\mathrm{i}} \mathrm{r}_{\mathrm{ij}}$, que indica el empleo generado en la región ante un incremento de un millón de pesetas en la demanda final de la rama $j$. Los resultados obtenido se presentan en el Cuadro 10.

En el mismo se observa cómo ambos indicadores, tanto para las ramas del CAA como para el conjunto de la economía, han disminuido sensiblemente en los quince años considerados, debido al proceso de sustitución del factor trabajo, de forma que se obtienen mayores producciones (expresadas en pesetas corrientes) con menos trabajadores.

Cuadro 10. Capacidad de generación de empleo.

\begin{tabular}{lcccccc}
\hline & \multicolumn{3}{c}{} & $\mathrm{p}_{\mathrm{i}}$ & & \multicolumn{3}{c}{$\mathbf{M E M P}_{\mathrm{j}}$} \\
\hline & 1980 & 1995 & $\mathrm{DIF}$ & 1980 & 1995 & $\mathrm{DIF}$ \\
\hline 1. Agricultura & 0,79 & 0,14 & $-0,65$ & 0,89 & 0,17 & $-0,72$ \\
2. Ganadería & 0,25 & 0,12 & $-0,13$ & 0,37 & 0,19 & $-0,18$ \\
3. Caza, silvicultura y pesca & 0,46 & 0,11 & $-0,35$ & 0,54 & 0,11 & $-0,43$ \\
Agricultura, ganadería y silvic. (1) & $\mathbf{0 , 5 0}$ & $\mathbf{0 , 1 2}$ & $\mathbf{- 0 , 3 8}$ & $\mathbf{0 , 6 0}$ & $\mathbf{0 , 1 6}$ & $\mathbf{- 0 , 4 4}$ \\
5. Industria de productos cárnicos & 0,15 & 0,03 & $-0,12$ & 0,36 & 0,10 & $-0,26$ \\
6. Fabricación de conservas vegetales & 0,35 & 0,07 & $-0,28$ & 0,65 & 0,15 & $-0,50$ \\
7. Industria de productos lácteos & 0,10 & 0,03 & $-0,07$ & 0,22 & 0,10 & $-0,12$ \\
8. Pan, bollería y galletas & 0,28 & 0,10 & $-0,18$ & 0,38 & 0,14 & $-0,24$ \\
9. Otras industrias de alimentación & 0,08 & 0,03 & $-0,05$ & 0,32 & 0,09 & $-0,23$ \\
10. Industria vinícola & 0,19 & 0,03 & $-0,16$ & 0,32 & 0,10 & $-0,22$ \\
11. Otras industrias de bebida y tabaco & 0,15 & 0,02 & $-0,13$ & 0,22 & 0,05 & $-0,17$ \\
Industria Agroalimentaria (1) & $\mathbf{0 , 1 8}$ & $\mathbf{0 , 0 4}$ & $\mathbf{- 0 , 1 4}$ & $\mathbf{0 , 3 5}$ & $\mathbf{0 , 1 0}$ & $\mathbf{- 0 , 2 5}$ \\
Complejo Agroalimentario (1) & $\mathbf{0 , 2 8}$ & $\mathbf{0 , 0 7}$ & $\mathbf{- 0 , 2 1}$ & $\mathbf{0 , 4 3}$ & $\mathbf{0 , 1 2}$ & $\mathbf{- 0 , 3 1}$ \\
Economía Navarra (1) & 0,54 & 0,09 & $-0,45$ & 0,65 & 0,13 & $-0,52$ \\
\hline Fuente: TION-80 y TION-95. (1) Los valores presentados son las medias de las ramas incluidas en cada agrupación, excepto en el caso del \\
multiplicador de empleo obtenido para el sector primario. & & & & &
\end{tabular}


También se desprende de la información disponible que tanto en 1980 como en 1995, es el sector primario el que más capacidad, directa e indirectamente, tiene de generar empleo ${ }^{13}$. Las ramas de fabricación de conservas vegetales y pan, bollería y galletas también generan más empleo que la media del CAA cuando aumentan sus demandas finales. De todas formas, mientras en 1980 la media del CAA era inferior a la del conjunto de la economía, en 1995 es similar a la media regional, debido a que esta capacidad ha disminuido más en el conjunto de la economía regional.

\section{CONCLUSIONES}

A la vista del análisis realizado del CAA a partir de la información suministrada por las TION-80 y TION-95, las conclusiones más relevantes son las siguientes:

En primer lugar, cabe destacar que las diez ramas de actividad que se incluyen en el CAA representan en Navarra una parte importante de la actividad económica, dadas sus elevadas participaciones en las macromagnitudes regionales, aunque en el período considerado han perdido importancia relativa.

Si consideramos como magnitudes más relevantes el VABpm y la producción efectiva, las actividades más importantes en el interior del complejo son la agricultura y la fabricación de conservas vegetales. La evolución de ambas ha sido diferente, ya que mientras la agricultura ha perdido relevancia, la fabricación de conservas vegetales, junto con la industria vinícola y las otras industrias de bebidas y tabaco han aumentado sensiblemente su participación en el CAA regional.

En cuanto a la articulación del CAA, cabe destacar el papel de la agricultura y ganadería como oferentes de inputs intermedios al resto del complejo, aunque en el período considerado su comportamiento ha sido dispar, ya que mientras la primera aumenta su importancia, la segunda la está perdiendo. Las ramas de fabricación de conservas vegetales, otras industrias de alimentación y la industria vinícola destacan por sus demandas de inputs intermedios. La ganadería también es importante a este respecto, con un sensible aumento en el período considerado de su capacidad de arrastre en el entorno regional. Sin embargo, se observa como la industria de productos cárnicos ha perdido importancia en este sentido.

13 Este resultado es una consecuencia de que el coeficiente directo de empleo, utilizado en el cálculo del multiplicador, es anormalmente elevado para el sector primario, porque no contempla el subempleo. 
A pesar de no existir ninguna rama clave, en el sentido definido anteriormente, en los dos años considerados destaca el papel de la agricultura como receptora de los impulsos del sistema económico y la fabricación de conservas vegetales como impulsora. En todo caso, la evolución de ambas ha sido distinta, ya que mientras la agricultura ha visto disminuir su capacidad, la fabricación de conservas vegetales la ha aumentado.

La capacidad de generar renta, directa e indirectamente, en la región por parte de las actividades del CAA es similar a la media regional, aunque en el período considerado ha aumentado en una cuantía sensiblemente superior a la media de las 51 ramas consideradas en las TION. Además, el comportamiento de las ramas es muy dispar, ya que mientras las ramas del sector primario generan como media 0,89 unidades, la IAA genera solamente 0,58. Las primeras se sitúan por encima de la media regional, mientras que las segundas se sitúan por debajo. De todas formas, dentro de este grupo destacan por su mayor capacidad de generar renta las ramas de fabricación de conservas vegetales y la de pan, bollería y galletas. Entre ellas, destacan por un aumento muy apreciable en los quince años considerados de su capacidad de generar renta la ganadería y la industria vinícola.

En cuanto a la capacidad para generar empleo, a partir de los indicadores obtenidos se detecta una sensible reducción en el conjunto de la economía. En todo caso, mientras en 1980 la media del CAA era inferior a la global, en 1995 es similar a la media regional debido al mayor ritmo de capitalización. Respecto a las ramas pertenecientes al CAA, los resultados son similares a los anteriores en el sentido de que el sector primario supera la media regional, mientras que la IAA no. Dentro de este conjunto destacan nuevamente las ramas de fabricación de conservas vegetales y la de pan, bollería y galletas.

\section{BIBLIOGRAFÍA}

Artis, M., Suriñach, J. y Pons, J. (1994). El sistema agroalimentario catalán en la tabla inputoutput de 1987. Investigación Agraria. Economía 9 (1): 53-75.

Cuadrado, J.R. y Mancha, T. (1984). Un análisis sobre los sectores polarizadores de la economía española. Información Comercial Española 607: 101-112.

Chenery, H. y Watanabe, T. (1958). International Comparisons of the structure of productions. Econometrica 56: 487-521.

De La Grana, C. y Azaceta, J. (1990). Aproximación al Sistema Agro-alimentario de la CA de Euskadi en base a técnicas Input-Output. En Eustat: Evolución de la Economía Vasca en el 
período 1980-85. Tablas Input-Output de la CA de Euskadi. Tomo III. Análisis de resultados. Zarautz (Guipúzcoa): 377-459.

De Pablo, J. y Céspedes, J. (1996). Análisis del complejo de producción agroalimentario andaluz a través de las tablas input-output (1980-1990). Revista Española de Economía Agraria 175 (1): 87-117.

Del Castillo, F. y García, M.V. (1990). Cambios en la articulación económica interna en la C. A. de Euskadi en el período 1980-85.. En Eustat: Evolución de la Economía Vasca en el período 1980-85. Tablas Input-Output de la CA de Euskadi. Tomo III. Análisis de resultados. Zarautz (Guipúzcoa): 150-233.

Del Castillo, F. y Martínez, J.M. (1986). Sobre la utilización de la matriz inversa de Leontief en economías abiertas. Estadística Española 112-113: 45-58.

Domingo, T., Pedreño, A., Fernández, I., Sanchís, M., García, L. y Suárez, C. (1987). Métodos y ejercicios de economía aplicada. Editorial Pirámide. Madrid

Enciso, J.P. y Sabaté, P. (1995a). Una visión del complejo de producción agroalimentario español en la década de los ochenta. Investigación Agraria. Economía 10 (3): 435-467.

Enciso, J.P. y Sabaté, P. (1995b). Los sectores agroalimentarios español y catalán, un análisis comparativo a través de la tabla input-output. Revista Española de Economía Agraria 171 (1): 53-83.

García Alvarez-Coque, J.M. y Enguídanos Weyler, M. (1999). El Sector Agroalimentario Valenciano en las Tablas Input-Output de la Comunidad Valenciana 1990. Generalitat Valenciana. Valencia.

Gobierno de Navarra (1997). Cuentas Regionales de Navarra. 1995. Tablas Input-Output. Gobierno de Navarra. Departamento de Economía y Hacienda. Servicio de Estadística. Pamplona.

Hirschman, A.O. (1958). The strategy of economic development. Yale University Press. New Heaven.

Iráizoz, B. y Rapún, M. (1999). El complejo agroalimentario de Navarra. Análisis a partir de las Tablas Input-Output de 1995. Revista de Estudios Regionales 55: 193-223.

López, M. (1995).Principales cambios estructurales de la industria Agroalimentaria española en la década de los ochenta.. Revista Española de Economía Agraria 171 (1): 9-51.

Malassis, L. (1979). Economie Agroalimentaire I. Economie de la consommation et de la production agroalimentaire. Cujas. Paris. 
Muñoz Cidad, C. (1988). Elaboración y utilización de las tablas input-output regionales. Papeles de Economía Española 35: 457-469.

Muñoz Cidad, C. (1994). Las cuentas de la nación. Nueva introducción a la economía aplicada. Cívitas. Colección Economía. Madrid.

Pérez y Pérez, L. y Feijoo, L. (1993). Estructura del complejo agro-alimentario aragonés a través de las tablas input-output. Revista de Estudios Agro-Sociales 164: 61-74.

Pulido, A y Fontela, E. (1993). Análisis Input-Output. Modelos, Datos y Aplicaciones. Pirámide. Madrid.

Rasmussen, P.N. (1956). Studies in Intersectorial Relations. north-Holland. Amsterdan.

Rodríguez Nuño, V. (1998). Cambios en el modelo de especialización sectorial del País Vasco y de las restante comunidades autónoomas en el período 1980-1994. Ekonomiaz 40: 248-269.

Streit, M.E. (1969). Spatial Asociations and Economic Linkages between industries. Journal of Regional Science 9 (2): 177-188.

Titos, A. (1974). El complejo industrial alimentario de Córdoba. Economía Industrial 122: 69-75.

Titos, A. (1995). El complejo agroalimentario andaluz: un análisis a partir de la TIOAN-90, en Instituto de Estadística de Andalucía: Contabilidad Regional y Tablas Input-Output de Andalucía 1990, vol. 2: 330-375

Titos, A. y De Haro, T. (1983). El complejo de producción agroalimentaria. Papeles de Economía Española 16: 22-37.

Titos, A., De Haro, T., Gómez, A. C. y Ramos, F. (1995). El sistema agroalimentario español. Tabla input-output y análisis de las relaciones intersectoriales. Ministerio de Agricultura, Pesca y Alimentación. Serie Estudios. Madrid.

Titos, A., De Haro, T. y Parra, C. (1996). Cambio estructural en el sistema agroalimentario español (1970/88). Publicaciones ETEA. Colección Monografías. Córdoba. 